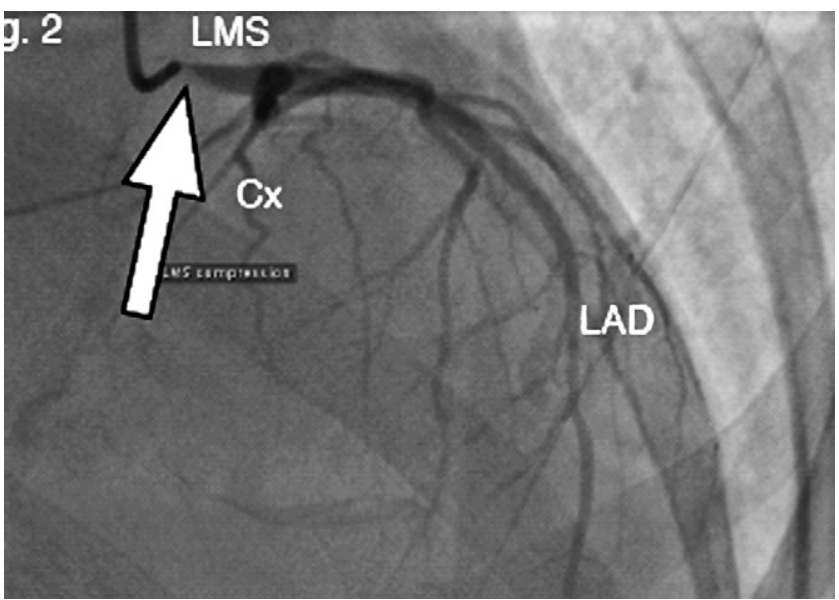

Figure 1. Coronary angiogram showing severe ostial left main coronary artery compression (white arrow). Cx, Circumflex artery; $L A D$, left anterior descending artery; $L M S$, left main stem coronary artery.

This case presents a rare cause of potentially fatal acute cardiac ischemia.

\section{References}

1. Kennebeck GA, Wong AK, Berry WR, Higgins JP, Manubens SM. Mediastinal bronchogenic cyst manifesting as catastrophic myocardial infarction. Ann Thorac Surg. 1999;67:1789-91.

2. Nomori H, Kameda T, Kobayashi R, Morinaga S. A case of intra-pericardial bronchogenic cyst requiring an emergency operation. Nippon Kyobu Geka Gakki Zasshi. 1993;41:452-5.

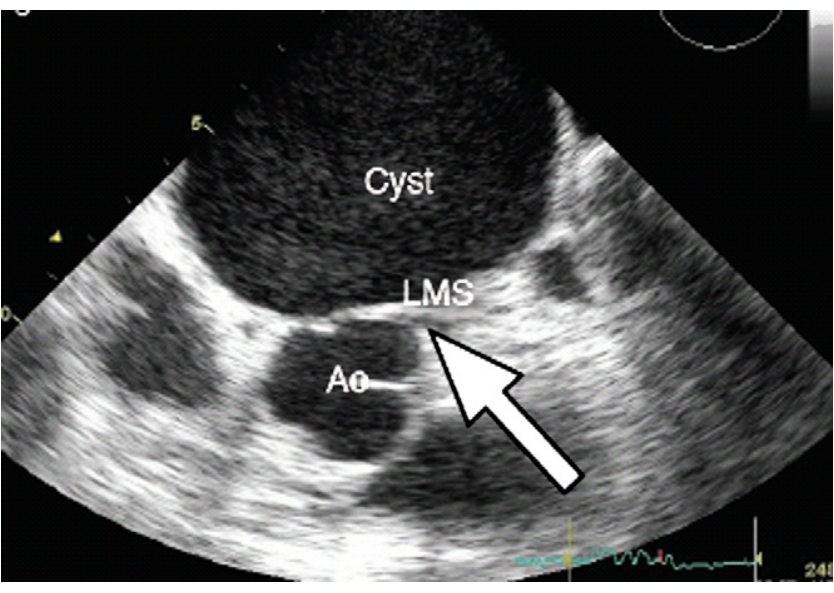

Figure 2. Perioperative TEE. Midesophageal short-axis view showing bronchogenic cyst compressing the left main stem coronary artery (LMS, white arrow).

\title{
Congenital Bochdalek hernia presenting with acute pancreatitis in an adult
}

Deborah K. Harrington, MD, MRCS, Frank T. Curran, MD, FRCS, Ian Morgan, MD, FRCS (CTh), and Patrick Yiu, PhD, FRCS (CTh), Wolverhampton, United Kingdom

\footnotetext{
From the Department of Cardiothoracic Surgery, Newcross Hospital, Royal Wolverhampton NHS Trust, Wolverhampton, United Kingdom.

Received for publication Nov 27, 2007; accepted for publication Jan 13, 2008.

Address for reprints: Deborah K. Harrington, MD, MRCS, Department of Cardiothoracic Surgery, Newcross Hospital, Royal Wolverhampton NHS Trust, Wolverhampton, West Midlands WV10 0QP (E-mail: dkharrington@hotmail.com).

J Thorac Cardiovasc Surg 2008;135:1396-7

$0022-5223 / \$ 34.00$

Copyright (C) 2008 by The American Association for Thoracic Surgery doi:10.1016/j.jtcvs.2008.01.038
}

3. Hauber J, Rein J, Schaudig G, Allmendinger G, Sigel H. High grade coronary stenosis due to bronchogenic cyst. Dtsch Med Wochenschr. 1995; 120:597-602

4. Lugo-Olivieri $\mathrm{CH}$, Schwartzman GJ, Fishman EK. Intrapericardial bronchogenic cyst: assessment with magnetic resonance imaging and transesophageal echocardiography. Clin Imag. 1999;2381-4.

5. St-Georges R, Deslauriers J, Duranceau A, Vaillancourt R, Deschamps C, Beauchamp G, et al. Clinical spectrum of bronchogenic cysts of the mediastinum and lung in the adult. Ann Thorac Surg. 1991; 52:6-13. 


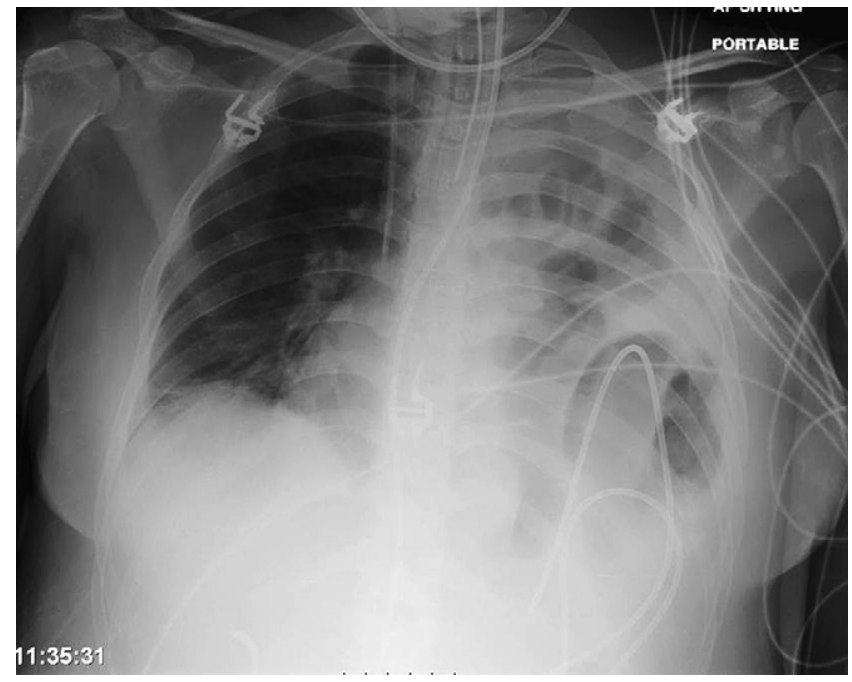

Figure 1. Chest x-ray revealing loops of bowel in the left side of the chest.

the left side of the chest. Her initial serum amylase was $778 \mathrm{U} / \mathrm{L}$, and chest X-ray revealed loops of bowel in the lower left side of the chest (Figure 1). The patient was treated with intravenous fluids, nasogastric aspiration, and analgesia; however, during the next 2 days, she became increasingly dyspnoeic requiring intubation and ventilation. A chest and abdominal computed tomography scan revealed acute pancreatitis with ascites, a large left pleural effusion with collapsed left lung, and a left-sided diaphragmatic hernia but no evidence of intestinal ischemia or perforation. After multidisciplinary discussion, a plan was made to treat the patient conservatively by pigtail drainage of the ascites and chest drainage of the left pleural collection, which also had a high amylase content. Initially, the patient made good progress, with reexpansion of the left lung and successful extubation; however, her condition again deteriorated requiring reventilation, and she continued to have large nasogastric aspirates. A further computed tomography scan revealed volvulus and distension of the stomach, which was treated by immediate endoscopic deflation. After failure of conservative treatment, urgent repair of the diaphragmatic hernia was then planned.

A left thoracolaparotomy was performed revealing a large posterolateral diaphragmatic hernia and a collapsed but normal left lung. The stomach, omentum, transverse colon, and loops of small intestine were found in the left side of the chest. The patient was also noted to have congenital asplenia. The contents of the hernia were reduced into the abdomen, and the defect was repaired using a composite Prolene/polytetrafluoroethylene mesh patch (Figure 2).

The patient had a good postoperative recovery and was extubated on day 2. After a period of postoperative ileus, she was discharged. The postoperative chest $\mathrm{x}$-ray findings were normal.

\section{Discussion}

The incidence of Bochdalek hernias is said to be from 1 in 2200 to 12,500 live births, and they are more common on the left side. ${ }^{3}$ They result in a posterolateral defect caused by incomplete muscular for-

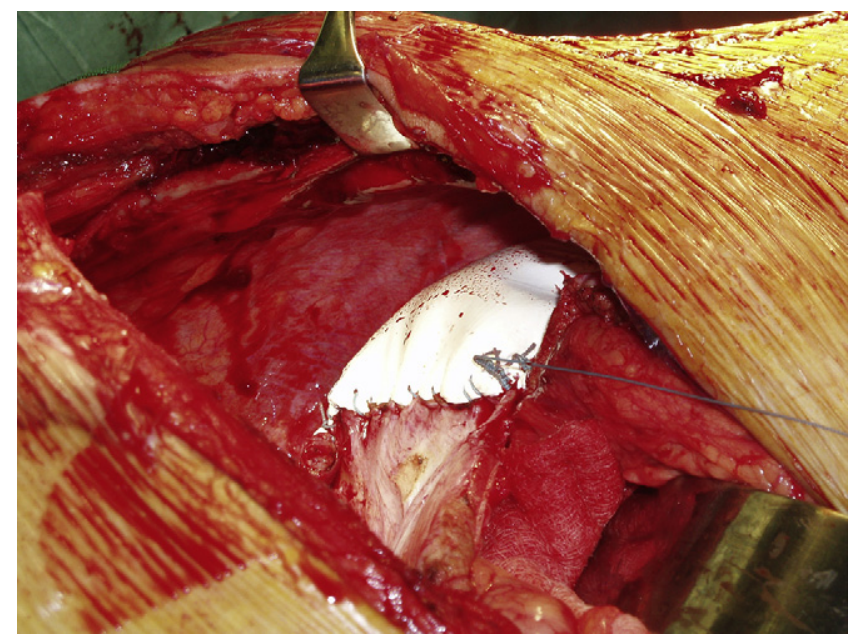

Figure 2. Photograph of diaphragmatic repair using Prolene/polytetrafluoroethylene mesh patch.

mation of the diaphragm in the region where the pleuroperitoneal membrane and septum transversum fuse. ${ }^{1}$

Although Bochdalek hernias are uncommon, adults presenting with complications of Bochdalek hernia, such as strangulation, incarceration, or volvulus of the stomach, are well documented. A presentation with acute pancreatitis, however, is rare, and we found few reports. In one case, a gastric volvulus in a Bochdalek hernia included the tail of the pancreas resulting in pancreatitis. ${ }^{4}$ Our case, on the other hand, had a resemblance to a case reported by Cuschieri and Wilson, ${ }^{5}$ in that the pancreas itself was not found among the hernia contents during any stage. No other obvious cause for pancreatitis (eg, gallstones or alcohol abuse) was evident. We therefore speculate that the pancreatitis was caused by traction after acute distension of the stomach and subsequent volvulus. Perhaps congenital asplenia in our patient may have further predisposed her to this mechanism.

We initially tried to reduce the hernia and treat type 2 respiratory failure by drainage of pleural and ascitic fluid, together with positive pressure ventilation. This conservative approach failed. Thus, we believe that acute pancreatitis in a setting of a diaphragmatic hernia with respiratory compromise is an indication for urgent repair.

Precise timing of surgery should be carefully reviewed, as dictated by the clinical status of the patient. The risk of surgery may be high during the immediate phase of pancreatitis. Delaying surgery may prolong lung collapse with its associated sequelae and risk repeated bowel herniation. A multidisciplinary approach among the general surgeon, intensivist, and cardiothoracic surgeon is advised.

\section{References}

1. Sugg W, Roper C, Carlsson E. Incarcerated Bochdalek hernias in the adult. Ann Surg. 1964;160:847-51.

2. Gale M. Bochdalek hernia: prevalence and CT characteristics. Radiology. 1985;156:449-52.

3. Robb B, Reed M. Congenital diaphragmatic hernia presenting as splenic rupture in an adult. Ann Thorac Surg. 2006;81:e9-10.

4. Oliver M, Wilson A, Kapila L. Acute pancreatitis and gastric volvulus occurring in a congenital diaphragmatic hernia. J Pediatr Surg. 1990;25:1240-1.

5. Cuschieri R, Wilson W. Incarcerated Bochdalek hernia presenting as acute pancreatitis. Br J Surg. 1981;68:669. 